\title{
Thermodynamic geometry of AdS black holes and black holes in a cavity
}

\author{
Peng Wang ${ }^{\mathrm{a}}$, Houwen $\mathrm{Wu}^{\mathrm{b}}$, Haitang Yang ${ }^{\mathrm{c}}$ \\ Center for Theoretical Physics, College of Physics, Sichuan University, Chengdu 610064, China
}

Received: 10 December 2019 / Accepted: 23 February 2020 / Published online: 6 March 2020

(C) The Author(s) 2020

\begin{abstract}
The thermodynamic geometry has been proved to be quite useful in understanding the microscopic structure of black holes. We investigate the phase structure, thermodynamic geometry and critical behavior of a ReissnerNordstrom-AdS black hole and a Reissner-Nordstrom black hole in a cavity, which can reach equilibrium in a canonical ensemble. Although the phase structure and critical behavior of both cases show striking resemblance, we find that there exist significant differences between the thermodynamic geometry of these two cases. Our results imply that there may be a connection between the black hole microstates and its boundary condition.
\end{abstract}

\section{Contents}

1 Introduction . . . . . . . . . . . . . 1

2 Phase structure and thermodynamic geometry . . . . 2

2.1 RN-AdS black holes . . . . . . . . . . 3

2.2 RN black holes in a cavity . . . . . . . . 6

3 Critical behavior ............. . . 8

4 Discussion and conclusion ........... . 10

References ................ 12

\section{Introduction}

The study of black hole thermodynamics has been playing an increasingly prominent role in our understanding of the interdisciplinary area of general relativity, quantum mechanics, information theory and statistical physics. In the pioneering work [1-3], black holes were found to possess thermodynamic properties such as entropy and temperature. Later,

\footnotetext{
a e-mail: pengw@scu.edu.cn

be-mail: iverwu@scu.edu.cn (corresponding author)

ce-mail: hyanga@scu.edu.cn
}

the Hawking-Page phase transition (i.e., a phase transition between the thermal anti-de Sitter (AdS) space and a black hole) was discovered in Schwarzschild-AdS black holes [4]. Unlike Schwarzschild black holes, Schwarzschild-AdS black holes can be thermally stable since the AdS boundary acts as a reflecting wall for the Hawking radiation. With the advent of the AdS/CFT correspondence [5-7], there has been much interest in studying the thermodynamics and phase structure of various AdS black holes [8-15]. Specifically, it was found that Reissner-Nordstrom-AdS (RN-AdS) black holes exhibit a van der Waals-like phase transition (i.e., a phase transition consisting of a first-order phase transition terminating at a second-order critical point) in a canonical ensemble $[9,10]$ and a Hawking-Page-like phase transition in a grand canonical ensemble [16].

Alternatively, one can make asymptotically flat black holes thermally stable by placing them inside a cavity, on the wall of which the metric is fixed. York first showed that Schwarzschild black holes in cavity can be thermally stable and have quite similar phase structure and transition to these of Schwarzschild-AdS black holes [17]. For ReissnerNordstrom (RN) black holes in a cavity, the thermodynamics and phase structure have been studied in a grand canonical ensemble [18] and a canonical ensemble [19,20]. It also showed that the phase structure of RN black holes in a cavity and RN-AdS black holes has extensive similarities. The phase structure of various black brane systems in a cavity was investigated in [21-26], and most of the systems were found to undergo Hawking-Page-like or van der Waals-like phase transitions. Boson stars and hairy black holes in a cavity were considered in [27-30], which showed that the phase structure of the gravity system in a cavity is strikingly similar to that of holographic superconductors in the AdS gravity. Moreover, the thermodynamic and critical behavior of de Sitter black holes in a cavity were investigated in the extended phase space [31]. Recently, we studied Born-Infeld black holes enclosed in a cavity in a canonical ensemble [32] and a 
grand canonical ensemble [33], respectively, and found that their phase structure has dissimilarities from that of BornInfeld-AdS black holes.

However, although it is believed that a black hole does possess thermodynamic quantities and extremely interesting phase structure, the statistical description of the black hole microstates has not yet been fully understood. Even though a complete quantum gravity theory is still absent, there have been some attempts to understand microscopic structure of a black hole [34-36]. Specifically, the thermodynamic geometry method has led to many insights into microstructure of a black hole. Following the pioneering work by Weinhold [37], Ruppeiner [38] introduced a Riemannian thermodynamic entropy metric to describe the thermodynamic fluctuation theory and found a systematic way to calculate the Ricci curvature scalar $R$ of the Ruppeiner metric. Later, $R$ has been computed for various ordinary thermodynamic systems, such as ideal quantum gases [39], Ising models [40] and anyon gas [41]. It showed that there is a relation between the type of the interparticle interaction and the sign of $R: R>0$ implies a repulsive interaction (e.g. ideal Bose gas) while $R<0$ means an attractive interaction (e.g. ideal Fermi gas), and $R=0$ corresponds to no interaction (e.g. ideal gas). ${ }^{1}$ It has also been indicated that, at a critical point, $|R|$ diverges as the correlation volume for ordinary thermodynamic systems [38].

The Ruppeiner geometry was subsequently exploited to probe the microstructure of a black hole. Since the work of [42], thermodynamic geometry has been studied for various black holes [43-56]. The result of [57] showed that a RN black hole has a vanished $R$, suggesting it is a non-interacting system. On the other hand, a Kerr-Newmann-AdS black can reduce to a RN black hole by making certain thermodynamic variables approach zero. In these limits, a RN black hole was found to acquire a nontrivial $R$ [58], implying that the phase space adopted in [57] may be incomplete. Recently, by choosing different thermodynamic coordinates, the authors of [59] showed that a RN black hole is an interaction system dominated by repulsive interaction. For a RN-AdS black hole, $R$ has been calculated, and it was observed that $R$ can be both positive and negative, and resembles the critical behavior of ordinary thermodynamic systems near a critical point $[45,51,57,60,61]$. The thermodynamic geometry has been investigated recently in the extended state space [62-73], in which the cosmological constant is treated as a thermodynamic variable and acts like a pressure term [74-76]. Inspired by the Ruppeiner geometry, a RN-AdS black hole was proposed to be built of some unknown micromolecules, inter-

\footnotetext{
1 We use the sign convention of Weinberg, where $R$ for the two-sphere is negative. Note that there is a different sign convention, where $R$ for the two-sphere is positive, used in the literature (e.g., [39]). So the sign of $R$ in [39] is opposite to that of our $R$.
}

actions among which can be tested by $R$ [64]. Recently, a new scalar curvature $R$ was introduced for a RN-AdS black hole, and it showed that there is a large difference between the microstructure of a black hole and the Van der Waals fluid [71].

Although there have been a lot of work in progress on thermodynamic geometry for various black holes of different theories of gravity in spacetimes with differing asymptotics, little is known about thermodynamic geometry for a black hole enclosed in a cavity. Unlike RN black holes, both RN-AdS black holes and RN black holes in a cavity can be thermally stable and hence provide an appropriate scenario to explore whether or not the thermodynamic geometry is sensitive to the boundary condition of black holes. In addition, it was recently proposed that the holographic dual of $T \bar{T}$ deformed $\mathrm{CFT}_{2}$ is a finite region of $\mathrm{AdS}_{3}$ with the wall at finite radial distance $[77,78]$, which further motivates us to investigate the properties of a black hole in a cavity. To this end, we undertake a study of the thermodynamic geometry for a RN black hole in a cavity. We report that, although the phase structure of a RN-AdS black hole and a RN black hole in a cavity is analogous to the van der Waals fluid, there are significant differences between the thermodynamic geometry of a RN-AdS black hole and that of a RN black hole in a cavity.

The rest of this paper is organized as follows. In Sect. 2, we first discuss the phase structure and thermodynamic geometry of a RN-AdS black hole in a canonical ensemble. Although the thermodynamic geometry in the thermodynamic coordinates of the charge $Q$ and potential $\Phi$ was investigated in $[45,51]$, we carry out the analysis in a more through way with a broader survey of the parameter space and find the $R>0$ region in the phase diagrams. The phase structure and thermodynamic geometry of a RN black hole in a cavity are then studied in details, starting with a discussion of its phase structure. In Sect. 3, the critical behavior of the RN-AdS black hole and the RN black hole in a cavity is obtained. We summarize our results with a brief discussion in Sect. 4. For simplicity, we set $G=\hbar=c=k_{B}=1$ in this paper.

\section{Phase structure and thermodynamic geometry}

In this section, we study phase structure and thermodynamic geometry of RN-AdS black holes and RN black holes in a cavity in a canonical ensemble. That said, the temperature and charge of the system are fixed. Thermodynamic geometry (Ruppeiner geometry) may provide a way to probe the microscopic structure of black holes. Adopting the Ruppeiner approach [38], one can define the Ruppeiner metric $g_{\mu \nu}^{R}$ for a thermodynamic system of independent variables $x^{\mu}$ as 
$g_{\mu \nu}^{R}=-\frac{\partial^{2} S(x)}{\partial x^{\mu} \partial x^{\nu}}$,

where $S$ is the entropy of the system. The Ruppeiner metric can be used to measure the distance between two neighboring fluctuation states [38]. More interestingly, the Ricci scalar of the Ruppeiner metric or the Ruppeiner invariant $R$ can shed light on some information about the microscopic behavior of the system, such as the strength and type of the dominated interaction between particles in the system.

\subsection{RN-AdS black holes}

The 4-dimensional static charged RN-AdS black hole solution is described by

$$
\begin{aligned}
d s^{2} & =-f(r) d t^{2}+\frac{d r^{2}}{f(r)}+r^{2}\left(d \theta^{2}+\sin ^{2} \theta d \phi^{2}\right), \\
A & =A_{t}(r) d t=-\frac{Q}{r} d t,
\end{aligned}
$$

where the metric function $f(r)$ is

$f(r)=1-\frac{2 M}{r}+\frac{Q^{2}}{r^{2}}+\frac{r^{2}}{l^{2}}$,

and $l$ is the AdS radius. The parameters $M$ and $Q$ can be interpreted as the black hole mass and charge, respectively. The Hawking temperature $T$ is given by sider the free energy. The free energy $F$ can be obtained by computing the Euclidean action in the semiclassical approximation and is given by

$F=M-T S$.

It was observed that the charge $Q$ and potential $\Phi$ of a RN-AdS black hole play similar roles as the pressure $P$ and volume $V$ of the van der Waals-Maxwell fluid in terms of determining the phase structure $[45,51]$. The correspondence $(\Phi, Q) \rightarrow(V, P)$ can establish the phase structure of the RN-AdS black hole. In [45], it was also suggested that the appropriate internal energy $U$ of the RN-AdS black hole is given by

$U=M-Q \Phi$,

where the contribution of the static electricity to the black hole mass $M$ is excluded. By analogy with the van der Waals fluid, we consider the parameter space coordinates $x^{\mu}=$ $(U, \Phi)$. Therefore, the Ruppeiner metric becomes

$g_{\mu \nu}^{R} d x^{\mu} d x^{\nu}=d U d\left(\frac{1}{T}\right)-\frac{Q}{T^{2}} d \Phi d T+\frac{1}{T} d Q d \Phi$.

Using Eqs. (4) and (5), we find that the expression of the Ruppeiner invariant $R$ in terms of the horizon radius $r_{+}$and the charge $Q$ is

$R=-\frac{\left(Q^{2}-r_{+}^{2}\right)^{2}+3 r_{+}^{2}\left(10 Q^{4}-9 Q^{2} r_{+}^{2}+3 r_{+}^{4}\right) / l^{2}+18 r_{+}^{6}\left(3 Q^{2}-r_{+}^{2}\right) / l^{4}}{\pi\left(r_{+}^{2}-Q^{2}+3 r_{+}^{4} / l^{2}\right)\left(3 Q^{2}-r_{+}^{2}+3 r_{+}^{4} / l^{2}\right)^{2}}$.

$T=\frac{1}{4 \pi r_{+}}\left(1-\frac{Q^{2}}{r_{+}^{2}}+\frac{3 r_{+}^{2}}{l^{2}}\right)$,

where $r_{+}$is the radius of the outer event horizon. Since $f\left(r_{+}\right)=0$, the mass $M$ can be expressed in terms of $r_{+}$:

$M=\frac{r_{+}}{2}\left(1+\frac{Q^{2}}{r_{+}^{2}}+\frac{r_{+}^{2}}{l^{2}}\right)$.

It can show that the RN-AdS black satisfies the first law of thermodynamics

$d M=T d S+\Phi d Q$,

where $S=\pi r_{+}^{2}$ and $\Phi=Q / r_{+}$is the entropy and potential of the black hole, respectively. To study the phase structure of the black hole in a canonical ensemble, we need to con-
We plot $R$ against $r_{+}$and $Q$ in the left panel of Fig. 1, where $R>0$ and $R<0$ in the yellow and green regions, respectively. The RN-AdS black hole solution in the gray region has negative temperature and hence is discarded. On the red lines, one has $R=-\infty$. The red line separating the "No BH" region and the " $R<0$ " region is determined by $T=0$, which shows that $R=-\infty$ for extremal RN-AdS black holes. The red line in the green region is given by $C_{Q}^{-1}=0$, where $C_{Q}$ is the heat capacity at constant $Q$ :

$C_{Q}=\left.T \frac{\partial S}{\partial T}\right|_{Q}=\frac{2 \pi r_{+} T}{\partial T /\left.\partial r_{+}\right|_{Q}}=\frac{8 \pi^{2} r_{+}^{5} T}{3 Q^{2}-r_{+}^{2}+3 r_{+}^{4} / l^{2}}$.

The divergence of $C_{Q}$ usually means that the black hole would undergo a phase transition. Moreover, using the mass representation of Ruppeiner metric, the authors of [80-82] showed that there is a one to one correspondence between 
Fig. 1 The Ruppeiner invariant $R$ as a function of the outer horizon radius $r_{+}$and the charge $Q$. Left Panel For a RN-AdS black hole, where $l$ is the AdS radius. Right Panel For a RN black hole in a cavity, where is $r_{B}$ the radius of the cavity. In the green (yellow) regions, $R<0$ $(R>0)$. The red lines correspond to $R=-\infty$. The black dots denote the critical points, which lie on the $R=-\infty$ lines. Black hole solutions do not exist in the gray regions

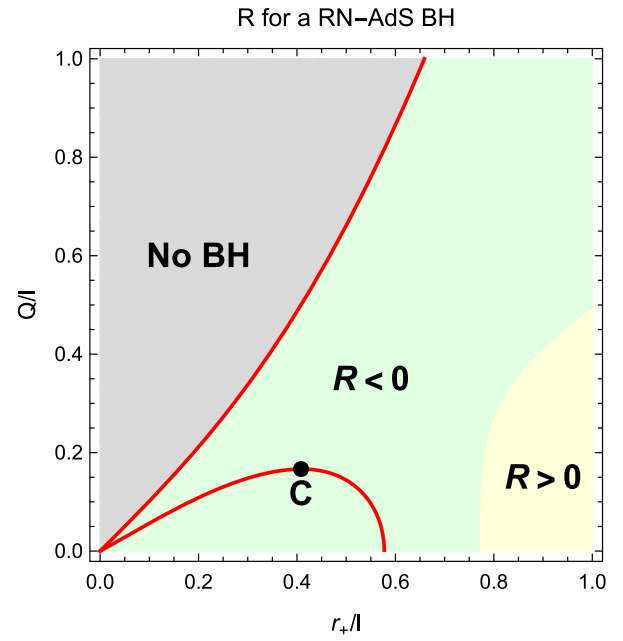

singularities of the Ruppeiner invariant and phase transitions of $C_{Q}$. For simplicity, we hereafter set $l=1$.

To study the Ruppeiner invariant $R$ as a function of the temperature $T$ and the charge $Q$, we need to use Eq. (4) to express the horizon radius $r_{+}$in terms of the temperature $T$ : $r_{+}=r_{+}(T)$. If $r_{+}(T)$ is multivalued, there is more than one black hole solution for fixed values of $Q$ and $T$, corresponding to multiple phases in the canonical ensemble. The critical point is an inflection point and obtained by

$$
\begin{aligned}
& \frac{\partial T}{\partial r_{+}}=0 \text { and } \frac{\partial^{2} T}{\partial r_{+}^{2}}=0 \\
& \quad\left(\text { or equivalently } \frac{\partial Q}{\partial \Phi}=0 \text { and } \frac{\partial^{2} Q}{\partial \Phi^{2}}=0\right),
\end{aligned}
$$

which gives the corresponding quantities evaluated at the critical point

$$
\left(r_{+c}, T_{c}, Q_{c}, \Phi_{c}\right) \approx(0.408,0.260,0.167,0.408)
$$

When $Q<Q_{c} \approx 0.167$, three black hole solutions coexist for some range of $T$. We plot $F$ and $R$ against $T$ for $Q=0.11$ in the left column of Fig. 2, which shows that there are three black hole solutions, dubbed as Large BH, Small BH and Intermediate $\mathrm{BH}$, when $T_{1}<T<T_{2}$. Note that since $\partial^{2} F / \partial^{2} T=-C_{Q}$, the thermally stable/unstable black hole solution has a concave downward/upward $T-F$ curve. So the upper-left panel of Fig. 2 shows that Large BH and Small BH are thermally stable while Intermediate BH is thermally unstable. It also displays that there is a first-order phase transition between Small BH and Large BH occurring at $T=T_{p}$ with $T_{1}<T_{p}<T_{2}$. So the globally stable phase is Large $\mathrm{BH}$ when $T>T_{p}$ and Small BH when $T<T_{p}$. As shown in the lower-left panel of Fig. 2, $R$ of Small BH and Intermediate $\mathrm{BH}$ is always negative, which means attractive interactions between the possible BH molecules. For Small $\mathrm{BH}, R=-\infty$ at $T=0$ and $T=T_{2}$, where $\left|C_{Q}\right|=\infty$. However for Large $\mathrm{BH}, R$ can be negative or positive depend- ing on the value of $T$. The inset shows that $R>0$ at a high enough temperature, and hence the interactions between the BH molecules become repulsive. It shows that $R$ of Large BH is negative infinity at $T=T_{1}$, where $\left|C_{Q}\right|=\infty$. Considering the globally stable phase, one has $R=-\infty$ only for the extremal black hole since Small BH at $T=T_{2}$ or Large $\mathrm{BH}$ at $T=T_{1}$ is not globally stable. There is a crossing of $R$ of Large BH and Small BH between $T_{1}$ and $T_{2}$. Such $R$-crossing was proposed to indicate a first-order phase transition due to the equality of the correlation lengths for the phases at the phase transition [79]. It is interesting to note that the temperature of the $R$-crossing is different from $T_{p}$. When $Q>Q_{c}$, there is only one black hole solution. We display $F$ and $R$ against $T$ for $Q=0.25$ in the right column of Fig. 2 . The upper-right panel of Fig. 2 shows that the black hole solution is always thermally stable. The lower-right panel of Fig. 2 shows that $R=-\infty$ at $T=0$, and $R>0$ when the temperature is high enough.

In Fig. 3, the globally stable phase of a RN-AdS black hole, which is the black hole solution with the minimum free energy, is displayed in the $Q-T$ space. There is a LBH/SBH first-order transition line for $Q<Q_{c}$, which terminates at the critical point. The Ruppeiner invariant $R$ is positive in the yellow region and negative infinity at $T=0$ and the critical point. Note that $R$ is always positive for a large enough value of $T$. Across the first-order transition line, the microstructure of the black hole changes while the type of the interaction among the microstructure is the same when $0.097 \lesssim Q<Q_{c}$. However, the type of the interaction changes across the transition line when $Q \lesssim 0.097$.

As discussed before, there is a correspondence $(\Phi, Q) \rightarrow$ $(V, P)$ for a RN-AdS and the van der Waals fluid when the phase structure is considered. To explore the resemblance between thermodynamics of these two systems, we investigate $Q$ and $R$ as functions of $T$ and $\Phi$. According to Fig. 3, there is no phase coexistence when $T>T_{c}$ or 

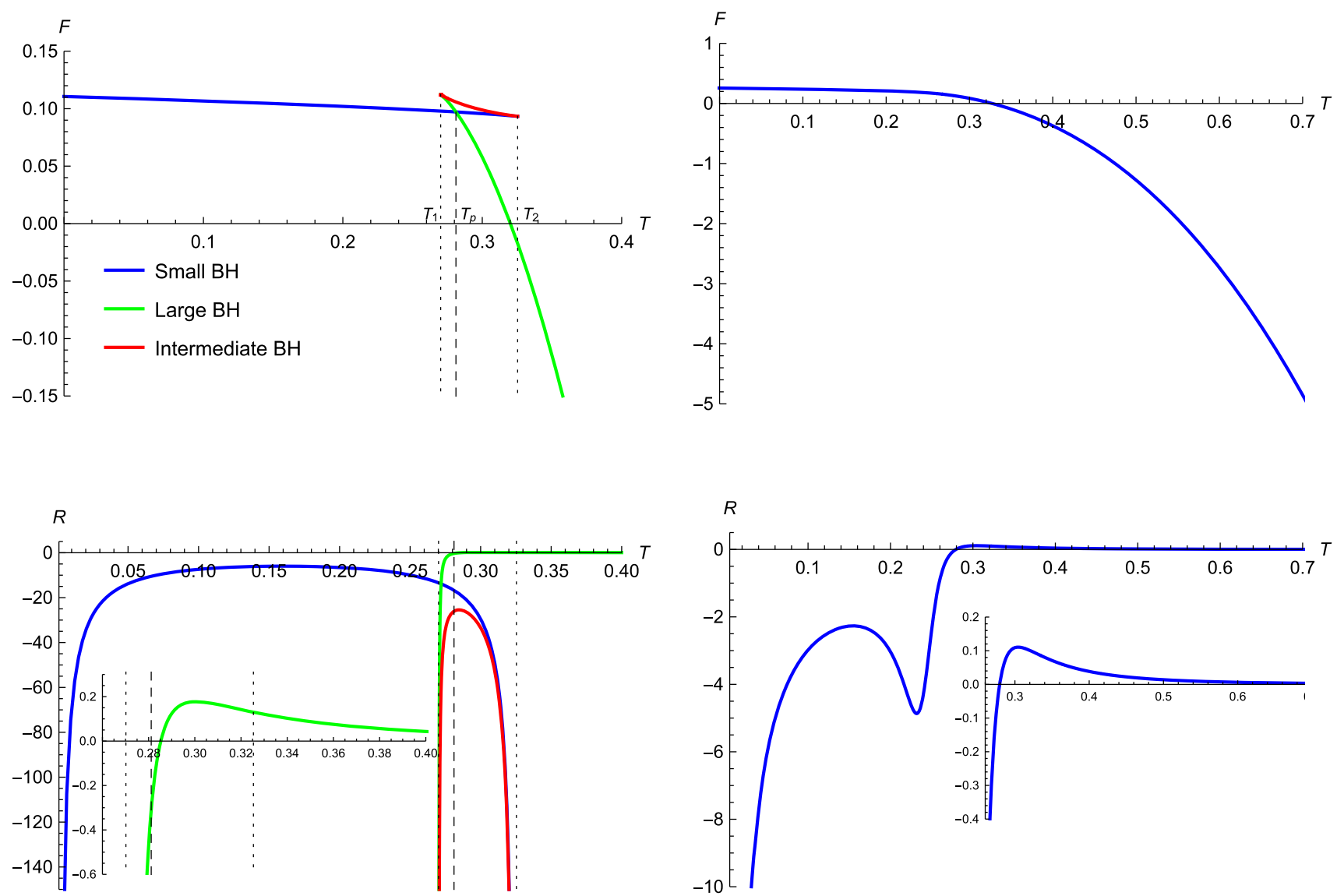

Fig. 2 The free energy $F$ and the Ruppeiner invariant $R$ as functions of the temperature $T$ for a RN-AdS black hole with a fixed charge $Q$. Left Column: $Q=0.11<Q_{c}$. There are three the black hole solutions when $T_{1}<T<T_{2}$. A LBH/SBH first-order phase transition occurs

$T<T_{0} \approx 0.318$, where $T_{0}$ is the temperature of the firstorder phase transition line at $Q=0$. For $T_{c}<T<T_{0}$, Large BH and Small BH can coexist for some range of $\Phi$ with some value of $Q$, which is determined by the first-order phase transition line (i.e., $F(\mathrm{LBH})=F(\mathrm{SBH})$ ). We plot $Q$ and $R$ versus $\Phi$ for $T=0.274$ in the left column of Fig. 4, where the black line is the coexisting line of Large BH and Small BH. Since Eq. (10) is not applicable on the coexisting line, we do not plot $R$ for the coexisting line. The lower-left panel of Fig. 4 shows that $R$ of Large $\mathrm{BH}$ is always negative and becomes divergent at the minimum value of $\Phi$, where $C_{Q}^{-1}=0$ and LBH is unstable and undercharged. For Small $\mathrm{BH}, R$ is negative and becomes divergent as $\Phi \rightarrow 1$. The unstable and overcharged Small BH also has $R=-\infty$ when $C_{Q}^{-1}=0$. The green and blue solid lines represent the globally stable phase, $R$ of which is always negative and becomes divergent as $\Phi \rightarrow 1$. We plot $Q$ and $R$ versus $\Phi$ for $T=0.3$ in the right column of Fig. 4. The Maxwell equal area rule is not applicable here since there is a jump of the free energy at $(\Phi, Q)=(0,0)$ on the isothermal $\Phi$ $Q$ line. When $\Phi$ is small enough, the inset shows that $R$ of

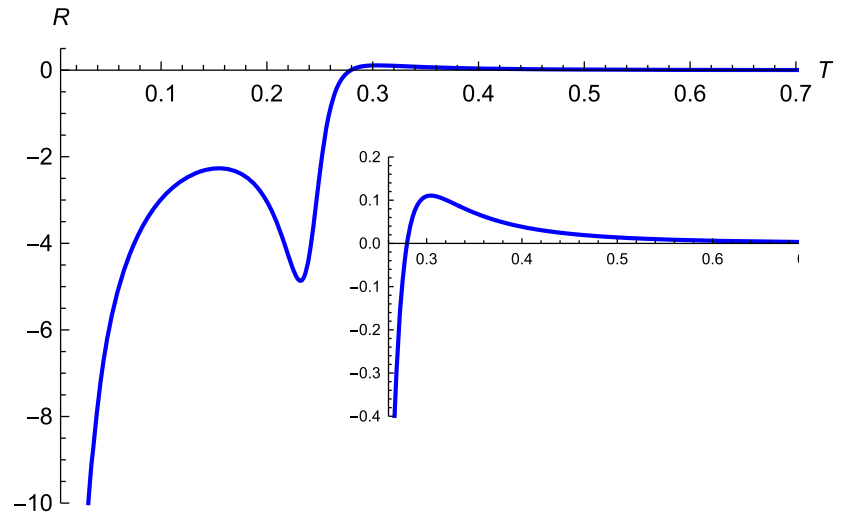

at $T=T_{p}$, and $R=-\infty$ at $T=T_{1}$ and $T_{2}$. The inset shows that $R>0$ for the Large BH solution at a high enough temperature. Right Column: $Q=0.25>Q_{c}$. There is only one black hole solution. The inset shows that $R>0$ when the temperature is high enough

Large $\mathrm{BH}$ becomes positive. For the globally stable phase, $R>0$ for a small enough $\Phi$ and $R \rightarrow-\infty$ as $\Phi \rightarrow 1$. Note that $\Phi \rightarrow 1$ corresponds to $M \rightarrow r_{+}\left(1-2 \pi r_{+} T\right)$ and $Q \rightarrow r_{+}\left(1-2 \pi r_{+} T\right)$ with $r_{+} \rightarrow 0$.

The globally stable phase of a RN-AdS black hole is plotted in the $\Phi-T$ space in Fig. 5. It is shown in Fig. 4 that, unlike the isothermal $V$ - $P$ lines in the van der Waals fluid, the isothermal $\Phi$ - $Q$ lines in the RN-AdS black hole are multivalued. In fact, there are two black hole solutions with different charges for fixed values of $\Phi$ and $T$. The left frame of Fig. 5 displays the large charge solution while the right one displays the small charge solution. Blow $T=T_{c}$, there is no phase coexistence, and only one globally stable phase, dubbed as Subcritical BH, exists. For Large BH, $R$ can be positive when $\Phi$ is small. As $\Phi \rightarrow 1, R$ of Small BH is negative infinity. When $T \lesssim 0.286$, the interactions among the microstructure of the saturated Large BH and Small BH on the coexisting line are both attractive. However when $T \gtrsim 0.286$, the interactions of the saturated Large $\mathrm{BH}$ and Small $\mathrm{BH}$ are repulsive and attractive, respectively. 


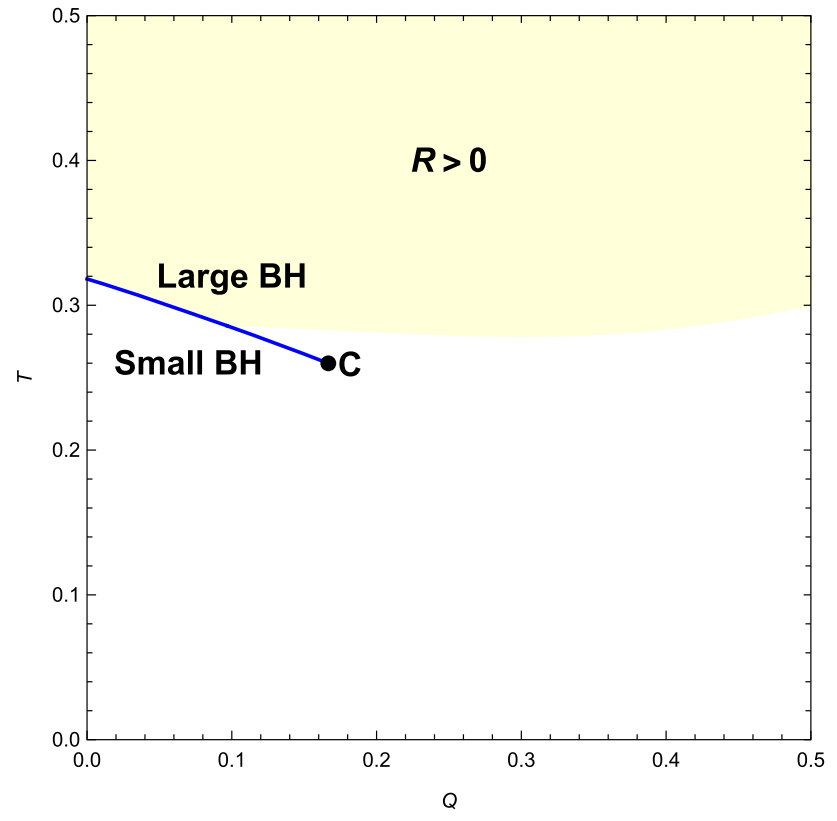

Fig. 3 Phase structure of a RN-AdS black hole in the $Q-T$ diagram. The first-order phase transition line separating Large $\mathrm{BH}$ and Small $\mathrm{BH}$ is displayed by the blue line and terminates at the critical point, marked by the black dot. In the yellow region, $R>0$

\subsection{RN black holes in a cavity}

The 4-dimensional RN black hole solution is

$$
\begin{aligned}
& d s^{2}=-f(r) d t^{2}+\frac{d r^{2}}{f(r)}+r^{2}\left(d \theta^{2}+\sin ^{2} \theta d \phi^{2}\right) \\
& f(r)=1-\frac{r_{+}}{r}+\frac{Q_{b}^{2}}{r^{2}}\left(1-\frac{r}{r_{+}}\right), A=A_{t}(r) d t=-\frac{Q_{b}}{r} d t
\end{aligned}
$$

where $Q_{b}$ is the black hole charge, and $r_{+}$is the radius of the outer event horizon. The Hawking temperature $T_{b}$ of the $\mathrm{RN}$ black hole is given by

$T_{b}=\frac{1}{4 \pi r_{+}}\left(1-\frac{Q_{b}^{2}}{r_{+}^{2}}\right)$ system, the free energy $F$ and the thermal energy $E$ were given in [19]

$$
\begin{aligned}
& F=r_{B}\left[1-\sqrt{f\left(r_{B}\right)}\right]-\pi T r_{+}^{2}, \\
& E=r_{B}\left[1-\sqrt{f\left(r_{B}\right)}\right] .
\end{aligned}
$$

It also showed in [18] that the system temperature $T$ and charge $Q$ can be related to the black hole temperature $T_{b}$ and charge $Q_{b}$ as

$$
\begin{aligned}
& Q=Q_{b}, \\
& T=\frac{T_{b}}{\sqrt{f\left(r_{B}\right)}},
\end{aligned}
$$

which means that $T$, measured at $r=r_{B}$, is blueshifted from $T_{b}$, measured at $r=\infty$. The potential $\Phi$ measured on the wall is [18]

$\Phi=\frac{A_{t}\left(r_{B}\right)-A_{t}\left(r_{+}\right)}{\sqrt{f\left(r_{b}\right)}}$

As in the RN-AdS black hole, we define the internal energy $U$ by excluding the contribution of the static electricity from the thermal energy $E$

$U=E-Q \Phi$

The physical space of $r_{+}$is bounded by

$r_{e} \leq r_{+} \leq r_{B}$

where $r_{e}=Q$ is the horizon radius of the extremal black hole.

In the parameter space coordinates $x^{\mu}=(U, \Phi)$, the Ruppeiner metric is

$g_{\mu \nu}^{R} d x^{\mu} d x^{\nu}=d U d\left(\frac{1}{T}\right)-\frac{Q}{T^{2}} d \Phi d T+\frac{1}{T} d Q d \Phi$,

and the Ruppeiner invariant $R$ as a function of the horizon radius $r_{+}$and the charge $Q$ is

$R=-\frac{\left(r_{+}^{2}-Q^{2}\right)\left(r_{+}-r_{B}\right)\left(Q^{2}-r_{+} r_{B}\right)\left[Q^{4}\left(12 r_{+}-5 r_{B}\right)-8 Q^{2} r_{+}^{2} r_{B}+r_{+}^{3} r_{B}\left(4 r_{B}-3 r_{+}\right)\right]}{\pi r_{+}^{2}\left[Q^{4}\left(6 r_{+}-5 r_{B}\right)-2 Q^{2} r_{+}\left(r_{+}^{2}+3 r_{+} r_{B}-3 r_{B}^{2}\right)+r_{+}^{3} r_{B}\left(3 r_{+}-2 r_{B}\right)\right]^{2}}$.

We now consider a thermodynamic system with a RN black holes enclosed in a cavity. Suppose that the wall of the cavity enclosing the RN black hole is at $r=r_{B}$, and the wall is maintained at a temperature of $T$ and a charge of $Q$. For this
We plot $R$ against $r_{+}$and $Q$ in the right panel of Fig. 1, where $R>0$ and $R<0$ in the yellow and green regions, respectively, and the solution in the gray region does not satisfy the constraint (20). The red line, on which $R=-\infty$, is also determined by $C_{Q}^{-1}=0$, where $C_{Q}$ is the heat capacity at constant $Q$ : 

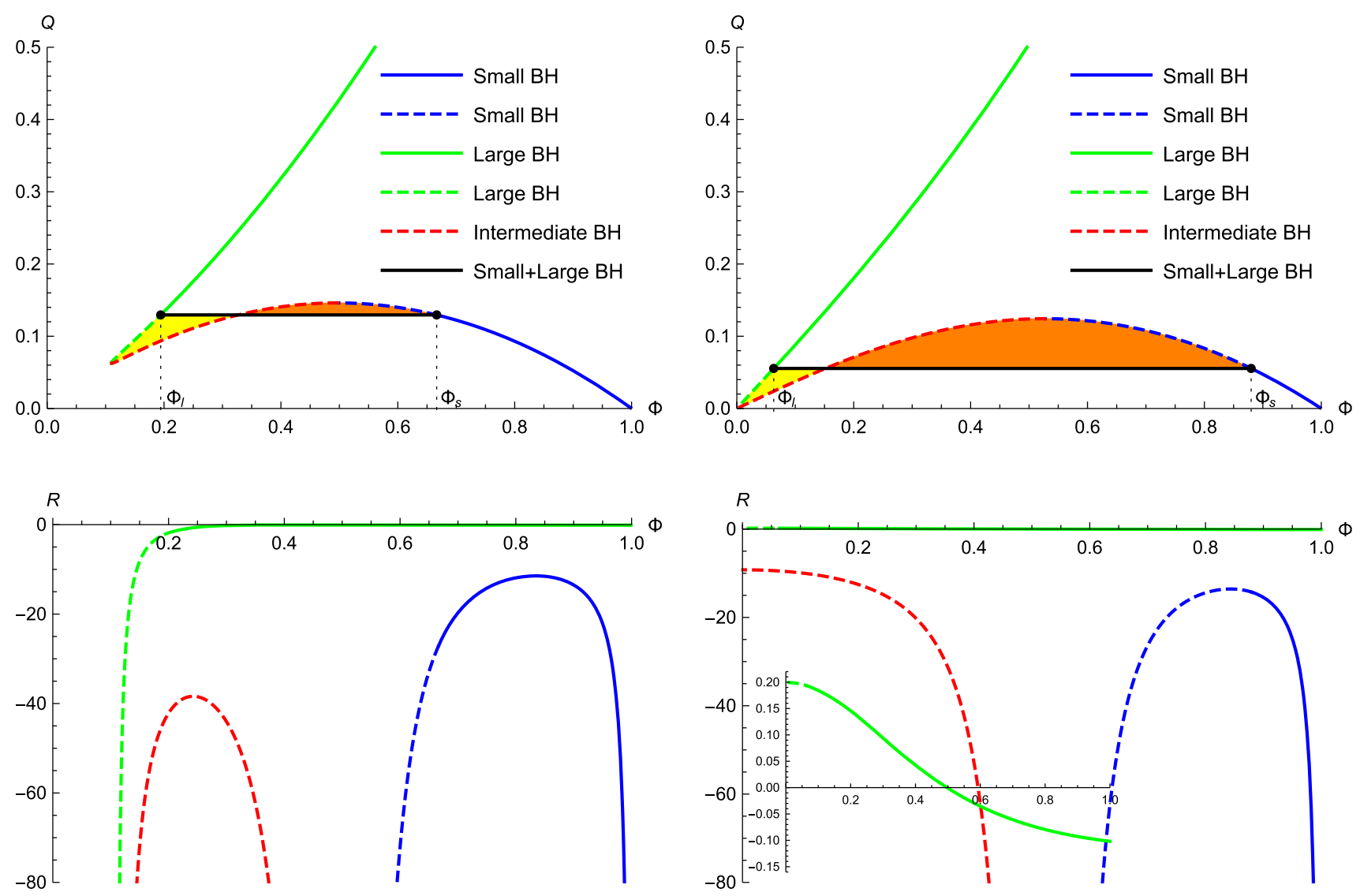

Fig. 4 The charge $Q$ and the Ruppeiner invariant $R$ as functions of the potential $\Phi$ for a RN-AdS black hole with a temperature $T>T_{c}$, which possesses three solutions for some range of $\Phi$. The $\Phi$ - $Q$ diagrams bear resemblance to that of the Van der Waals fluid. The coexisting lines of Large BH and Small BH are represented by the black lines, and $\Phi_{l} / \Phi_{s}$ denotes the potential of the saturated Small/Large BH. The blue/green dashed lines represent metastable overcharged Small BH/undercharged

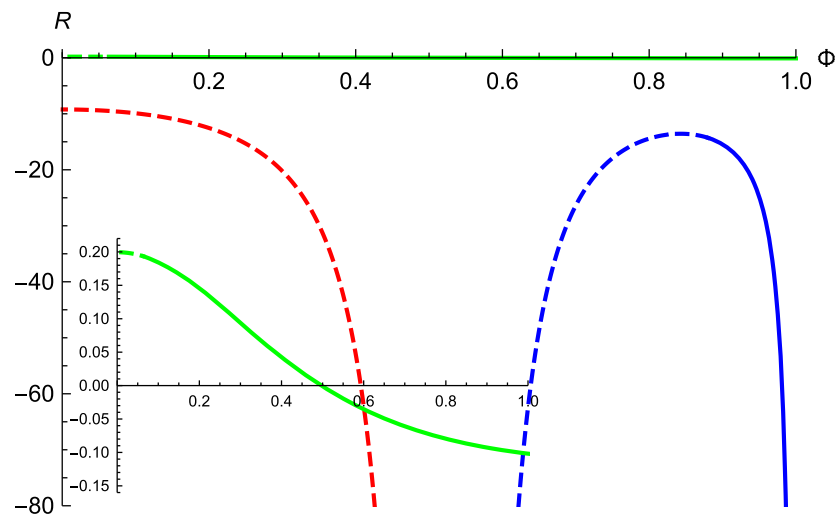

Large $\mathrm{BH}$ while the red dashed lines denote unstable spinodal Intermediate BH. Left Column $T=0.274$. The Maxwell equal area rule applies to determine the coexisting line, and $R$ is always negative. Right Column $T=0.3$. The Maxwell equal area rule does not apply to determine the coexisting line due to the discontinuity of the free energy at the origin. The inset shows that $R>0$ for the Large BH solution with a small enough potential

$C_{Q}=\frac{16 \pi^{2} T r_{+}^{9 / 2}\left(r_{B}-r_{+}\right)^{3 / 2}\left(r_{+} r_{B}-Q^{2}\right)^{3 / 2}}{r_{B}\left[Q^{4}\left(6 r_{+}-5 r_{B}\right)-2 Q^{2} r_{+}\left(r_{+}^{2}+3 r_{+} r_{B}-3 r_{B}^{2}\right)+r_{+}^{3} r_{B}\left(3 r_{+}-2 r_{B}\right)\right]}$.

Unlike the RN-AdS black hole, Eq. (22) gives $R=0$ for the extremal RN black hole with $Q=r_{+}$. Figure 1 shows that the behavior of $R$ of RN-AdS black holes is quite different from that of RN black holes in a cavity. For simplicity, we hereafter set $r_{B}=1$.

It was observed that the phase structure of a RN black hole in a cavity is strikingly similar to that of a RN-AdS black hole in the $Q-T$ space of a canonical ensemble [19,20]. Actually, a van der Waals-like phase transition occurs in both cases. Similar to the RN-AdS black hole, the RN black hole in a cavity possesses a critical point, which is determined by

$$
\frac{\partial T}{\partial r_{+}}=0 \quad \text { and } \quad \frac{\partial^{2} T}{\partial r_{+}^{2}}=0
$$

Solving the above equations gives quantities evaluated at the critical point

$\left(r_{+c}, T_{c}, Q_{c}, \Phi_{c}\right) \approx(0.528,0.186,0.236,0.325)$

The number of the solution(s) $r_{+}(T)$ to $T=T\left(r_{+}\right)$depends on the value of $Q$. When $Q<Q_{c} \approx 0.236$, there are three solutions coexisting for some range of $T$. We plot $F$ and $R$ against $T$ for $Q=0.15$ in the left column of Fig. 6, which shows that three solutions, dubbed as Large BH, Small BH and Intermediate $\mathrm{BH}$, coexist when $T_{1}<T<T_{2}$. Note that Large $\mathrm{BH}$ and Small $\mathrm{BH}$ are the thermally stable while Intermediate $\mathrm{BH}$ is thermally unstable. The system undergoes a first-order phase transition between Small BH and Large BH occurring at $T=T_{p}$ with $T_{1}<T_{p}<T_{2}$. The lower-left 


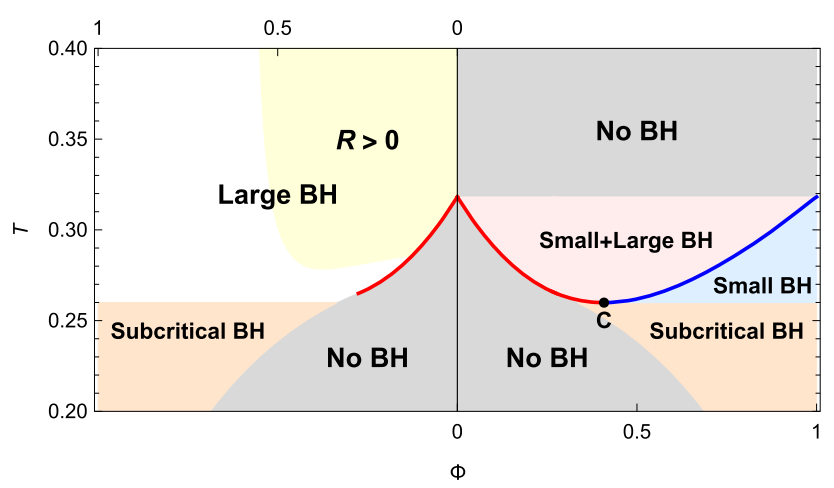

Fig. 5 Phase structure of a RN-AdS black hole in the $\Phi-T$ diagram. The critical point (black dot) separates the saturated Small BH and Large $\mathrm{BH}$, which are represented by the blue and red lines, respectively. Note that there are two black hole solutions for a fixed value of $\Phi$. The left/right frame shows the large/small $Q$ black hole solution. In the yellow region, $R>0$. The pink region is the coexistence region of Small BH and Large BH

panel of Fig. 6 and the inset display that $R$ of all three solutions is always negative, which means attractive interactions between the possible $\mathrm{BH}$ molecules. There is also a crossing of $R$ of Large BH and Small BH between $T_{1}$ and $T_{2}$. For Large $\mathrm{BH} / \mathrm{Small} \mathrm{BH}, R=-\infty$ at $T=T_{1} / T_{2}$, where $\left|C_{Q}\right|=\infty$. However, $R=0$ when $T=0$, which gives that $R$ of the globally stable phase is always finite. When $Q>Q_{c}$, there is only one solution. We depict $F$ and $R$ as functions of $T$ for $Q=0.4$ in the right column of Fig. 6. The upper-right panel of Fig. 6 shows that the solution is always thermally stable. The lower-right panel of Fig. 6 and the inset show that $R$ is always finite, and $R>0$ when the temperature is high enough or low enough.

In Fig. 7, the globally stable phase of a RN black hole in a cavity is displayed in the $Q-T$ space. There is a LBH/SBH first-order transition line for $Q<Q_{c}$, which terminates at the critical point. The Ruppeiner invariant $R$ is positive in the yellow region and negative infinity only at the critical point. Note that $R$ is always positive when $Q$ is large enough. Since $R$ of Large $\mathrm{BH}$ above and Small $\mathrm{BH}$ below the transition line is negative, the type of the interaction among the microstructure of the black hole stays the same when the system undergoes the first-order transition.

We now investigate the phase structure of a RN black hole in a cavity in the $\Phi-T$ space, which has not been discussed in the literature. Similar to a RN-AdS black hole, there is phase coexistence of Large BH and Small BH when $T_{c}<T<$ $T_{0} \approx 0.269$. We plot $Q$ and $R$ versus $\Phi$ for $T=0.2$ in the left column of Fig. 8, where the black line is the coexisting line of Large BH and Small BH. The upper bound on the $\Phi$ of Large BH comes from $r_{+} \leq 1$. The lower-left panel of Fig. 8 displays that $R$ of all three solutions is always negative and becomes divergent when $C_{Q}^{-1}=0$ or $\Phi \rightarrow 1$. Note that $\Phi \rightarrow 1$ corresponds to $Q \rightarrow r_{+}\left(1-2 \pi r_{+} T\right)$ with $r_{+} \rightarrow 0$.
We plot $Q$ and $R$ versus $\Phi$ for $T=0.3$ in the right column of Fig. 8. The inset shows that $R$ of Large BH is always finite and becomes positive when $\Phi$ is large enough. For Small BH, $R<0$ and $R \rightarrow-\infty$ as $\Phi \rightarrow 1$.

The globally stable phase of a RN-AdS black hole is plotted in the $\Phi-T$ space in Fig. 9, the left/right frame of which displays the large/small charge solution. There is Subcritical $\mathrm{BH}$ and no phase coexistence blow $T=T_{c}$. For Large BH, $R$ can be positive when $\Phi$ is large enough. As $\Phi \rightarrow 1, R$ of Small BH approaches negative infinity. Unlike the RN-AdS black hole case, the interactions among the microstructure of the saturated Large BH and Small BH on the coexisting line are always attractive.

\section{Critical behavior}

In this section, we investigate the thermodynamic behavior near the critical point. The critical behavior in a RN-AdS black hole has been discussed in [45,51], which showed that the critical exponents for a RN-AdS black hole and the Van der Waals fluid are identical. However, the critical exponents for a RN black hole in cavity have not been calculated yet.

First, we define

$t=\frac{T-T_{c}}{T_{c}} \quad$ and $\quad \phi=\frac{\Phi-\Phi_{c}}{\Phi_{c}}$.

In the neighborhood of the critical point, we can expand $Q$ in terms of $t$ and $\phi$

$Q=\sum_{i, j=0} q_{i j} t^{i} \phi^{j}$

where $q_{00}=Q_{c}$, and Eq. (12) gives $q_{01}=q_{02}=0$. Specifically for a RN-AdS black hole and a RN black hole in a cavity, we find

$$
\begin{aligned}
Q= & \frac{1}{6}-\frac{\phi^{3}}{3}+t\left(-\frac{2}{3}+2 \phi-8 \phi^{2}+\frac{28}{3} \phi^{3}\right)+\cdots, \\
Q= & 0.236-0.182 \phi^{3}+t(-0.618+1.118 \phi \\
& \left.-2.834 \phi^{2}+2.045 \phi^{3}\right)+\cdots,
\end{aligned}
$$

respectively. Near the critical point, the Maxwell equal area rule is applicable and gives

$\int_{\Phi_{s}}^{\Phi_{l}} \Phi d Q=0$ with $Q\left(\Phi_{l}\right)=Q\left(\Phi_{s}\right)$,

where $\Phi_{s}$ and $\Phi_{l}$ denote the potential of the saturated Small $\mathrm{BH}$ and Large BH, respectively. Substituting Eq. (27) into 

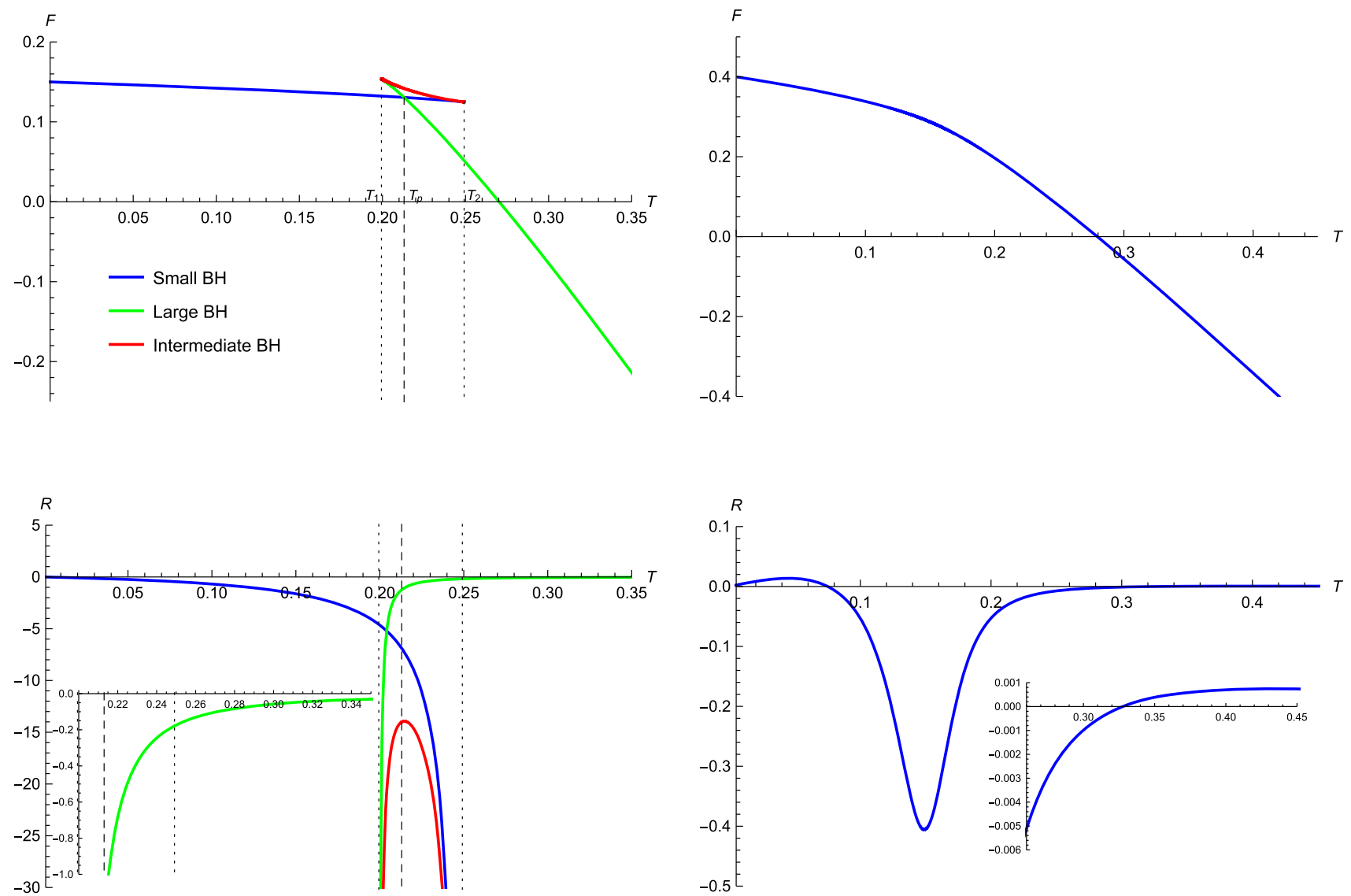

Fig. 6 The free energy $F$ and the Ruppeiner invariant $R$ as functions of the temperature $T$ for a RN black hole in a cavity with a fixed charge $Q$. Left Column $Q=0.15<Q_{c}$. Three black hole solutions coexist for $T_{1}<T<T_{2}$. A LBH/SBH first-order phase transition occurs at

$T=T_{p}$, and $R=-\infty$ at $T=T_{1}$ and $T_{2}$. The $T-R$ diagram and the inset show that $R$ is always negative. Right Column $Q=0.4>Q_{c}$. There is only one black hole solution. As shown in the $T-R$ diagram and the inset, $R>0$ when the temperature is high enough or low enough

Eq. (29), we have

$\phi_{l}=-\phi_{s}, \phi_{l}=\sqrt{-\frac{q_{11}}{q_{03}} t}+\mathcal{O}(t)$,

where $\phi_{l / s} \equiv\left(\Phi_{l / s}-\Phi_{c}\right) / \Phi_{c}$.

The critical exponents $\alpha, \beta, \gamma$ and $\delta$ are defined as follows:

- Exponent $\alpha$ describes the critical behavior of the specific heat at constant $\Phi: C_{\Phi} \propto|t|^{\alpha}$. We find that, at the critical point,

$C_{\Phi}=\left\{\begin{array}{c}-4.189 \text { for a RN-AdS BH, } \\ -3.167 \text { for a RN BH in a cavity, }\end{array}\right.$

which gives $\alpha=0$ in both cases.

- Exponent $\beta$ describes the critical behavior of the order parameter $\phi_{l}-\phi_{s}: \phi_{l}-\phi_{s} \propto|t|^{\beta}$. Equation (30) gives

$\phi_{l}-\phi_{s} \sim\left\{\begin{array}{c}4.899 \sqrt{t} \text { for a RN-AdS BH, } \\ 4.952 \sqrt{t} \text { for a RN BH in a cavity, }\end{array}\right.$

which leads to $\beta=1 / 2$ in both cases.

- Exponent $\gamma$ describes the critical behavior of the isothermal compressibility:

$\kappa_{T}=-\left.\frac{1}{\Phi} \frac{\partial \Phi}{\partial Q}\right|_{T} \propto|t|^{-\gamma}$.

Approaching the critical point along the coexistence curve, we use Eq. (27) to obtain

$\kappa_{T} \sim \frac{1}{2 q_{11} t} \sim\left\{\begin{array}{c}0.25 t^{-1} \text { for a RN-AdS BH, } \\ 0.447 t^{-1} \text { for a RN BH in a cavity, }\end{array}\right.$

which gives $\gamma=1$ in both cases.

- Exponent $\delta$ describes the critical behavior of $\left|Q-Q_{c}\right| \propto$ $|\phi|^{\delta}$ when $T=T_{c}$. On the critical isotherm $T=T_{c}$, Eq. (27) reduces to

$$
\begin{aligned}
\left|Q-Q_{c}\right| & \sim\left|q_{03}\right| \phi^{3} \\
& \sim\left\{\begin{array}{c}
\phi^{3} / 3 \text { for a RN-AdS BH, } \\
0.182 \phi^{3} \text { for a RN BH in a cavity, }
\end{array}\right.
\end{aligned}
$$




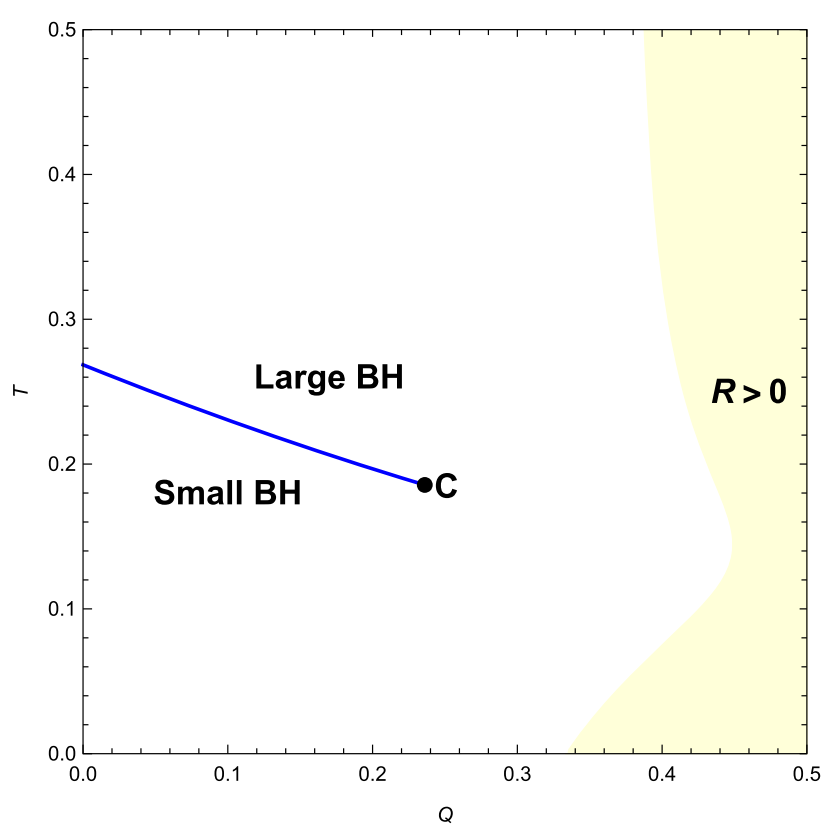

Fig. 7 Phase structure of a RN black hole in a cavity in the $Q-T$ diagram. The first-order phase transition line separating Large $\mathrm{BH}$ and Small $\mathrm{BH}$ is displayed by the blue line and terminates at the critical points (black dot). In the yellow region, $R>0$

which gives $\delta=3$ in both cases.

Our results show that the critical exponents of a RN black hole in cavity are also identical to these of the Van der Waals fluid predicted by the mean field theory. The critical exponents are believed to be universal since they are insensitive to the details of the physical system.

Finally, we study the critical behavior of the Ruppeiner invariant $R$ by expanding $R$ along the saturated Small BH and Large BH curves near the critical point. For the saturated Small BH and Large BH, we have

$r_{+l / s}-r_{+c} \simeq \frac{\Phi_{l / s}-\Phi_{c}}{\partial \Phi /\left.\partial r\right|_{Q=Q_{c}}} \simeq \pm \frac{\Phi_{c}}{\partial \Phi /\left.\partial r\right|_{Q=Q_{c}}} \sqrt{-\frac{q_{11}}{q_{03}} t}$

where $+/-$ is for the saturated Large $\mathrm{BH} / \mathrm{Small} \mathrm{BH}$, and $r_{+l / s}$ is the horizon radius of the saturated Large $\mathrm{BH} / \mathrm{Small}$ BH. In addition, the charge $Q$ for the coexisting line is given by

$Q \simeq Q_{c}+q_{03} \phi_{l / s}^{3}+q_{10} t+q_{11} t \phi_{l / s} \simeq Q_{c}+q_{10} t$.

Plugging Eqs. (35) and (36) into Eqs. (10) and (22), we can expand $R$ at $t=0$ and obtain

$R \sim\left\{\begin{array}{c}-0.030 t^{-2} \text { for a RN-AdS BH, } \\ -0.016 t^{-2} \text { for a RN BH in a cavity, }\end{array}\right.$ where the leading order terms of the expansions are the same for the saturated Small BH and Large BH. From Eqs. (31) and (37), we find

$\lim _{t \rightarrow 0} R C_{\Phi} t^{2}=\left\{\begin{array}{c}1 / 8 \text { for a RN-AdS BH, } \\ 0.051 \text { for a RN BH in a cavity }\end{array}\right.$

which shows that the critical value of $R C_{\Phi} t^{2}$ may depend on the boundary conditions of the black hole. In [71], $R$ of an RN-AdS black hole was calculated in the thermodynamic coordinates $x^{\mu}=(T, V)$, and it was found $\lim _{t \rightarrow 0} R C_{V} t^{2}=$ $-1 / 8$, which agrees with the numerical result of the Van der Waals fluid. However, there is a sign difference between our AdS result in Eq. (38) and the result in [71], which comes from $C_{\Phi}<0$ at the critical point for a RN-AdS black hole.

\section{Discussion and conclusion}

In this paper, we studied the phase structure, thermodynamic geometry and critical behavior of RN black holes in a canonical ensemble by considering two boundary conditions, namely the asymptotically AdS boundary and the Dirichlet boundary in the asymptotically flat spacetime. The phase structure of a RN-AdS black hole and a RN black hole in a cavity in the $Q-T$ space was displayed in Figs. 3 and 7, respectively, and the phase structure in the $\Phi-T$ space was shown in Figs. 5 and 9. It showed that the phase structure in both cases are quite similar. Specifically, with a correspondence $(\Phi, Q) \rightarrow(V, P)$, the phase structure of the RN-AdS black hole and the RN black hole in a cavity was rather similar to that of the Van der Waals fluid. We also calculated the critical exponents for the RN-AdS black hole and the RN black hole in a cavity and found they are identical to these of the Van der Waals fluid.

However, we found that the thermodynamic geometry in the AdS and cavity cases is quite different. The Ruppeiner invariant $R$ as a function of the horizon radius $r_{+}$and the charge $Q$ was obtained in the AdS and cavity cases and plotted in Fig. 1, which showed that the $R>0$ regions (yellow regions) are dissimilar in the two cases. Moreover, the extremal RN-AdS black hole has $R=-\infty$ while $R=0$ for the extremal RN black hole in a cavity. We also discussed $R$ as a function of $T$ and $Q$ and summarize the results for the AdS and cavity cases in Table 1 . Moreover, we found that the interactions among the microstructure of the black hole always stay attractive before and after the LBH/SBH first-order transition in the cavity case. However for a RNAdS black hole with $Q \lesssim 0.097$, the type of the interactions changes when the black hole undergoes the phase transition. The Ruppeiner invariant $R$ as a function of $\Phi$ and $T$ was also discussed, and it showed that $R \rightarrow-\infty$ as $\Phi \rightarrow 1$ with fixed $T$. Figures 5 and 9 displayed that Large $\mathrm{BH}$ with a small 

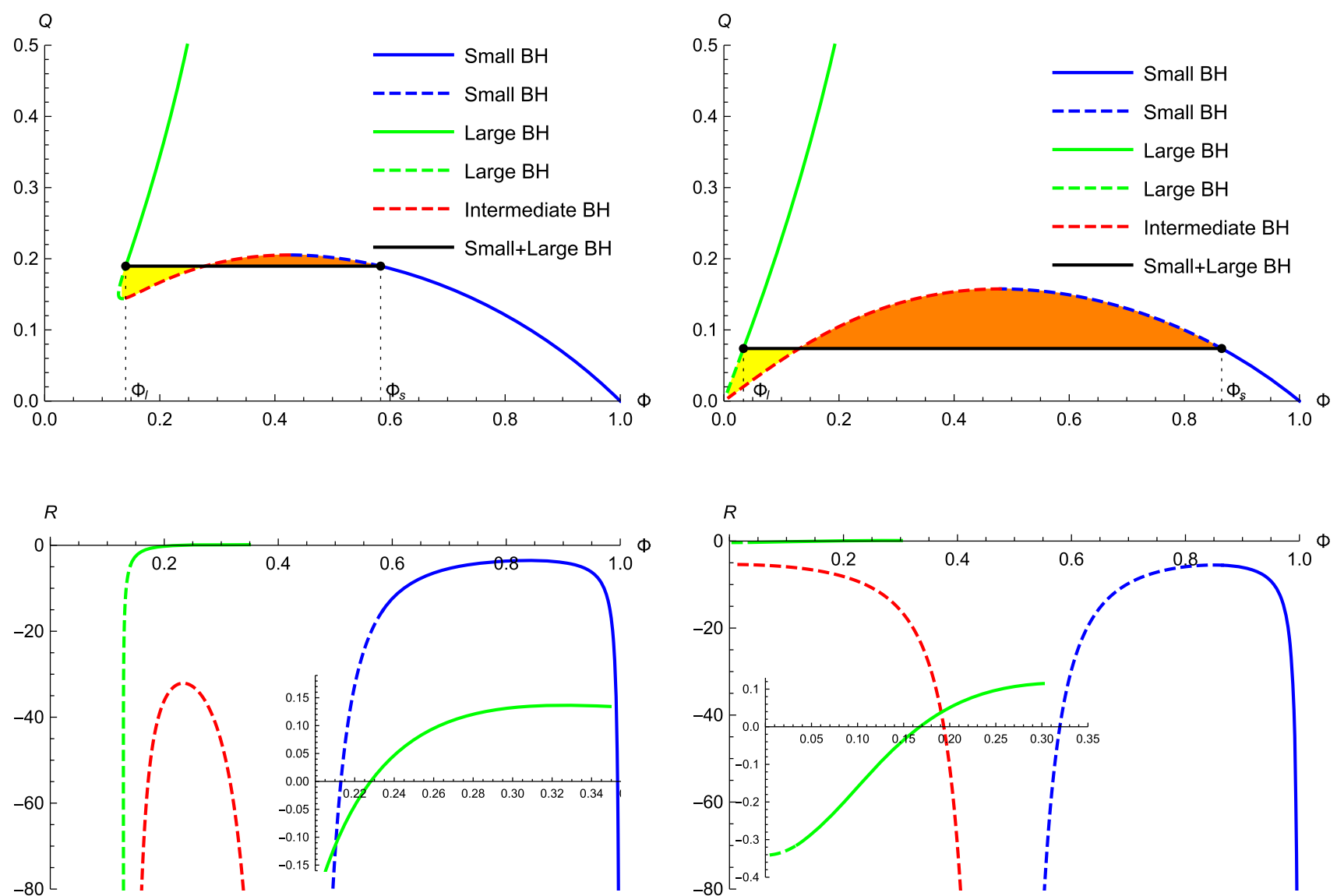

Fig. 8 The charge $Q$ and the Ruppeiner invariant $R$ as functions of the potential $\Phi$ for a RN black hole in a cavity with a temperature $T>T_{c}$, which possesses three solutions for some range of $\Phi$. The black lines denote the coexisting lines of Large $\mathrm{BH}$ and Small BH, and $\Phi_{s} / \Phi_{l}$ denotes the potential of the saturated Small/Large BH. The blue/green

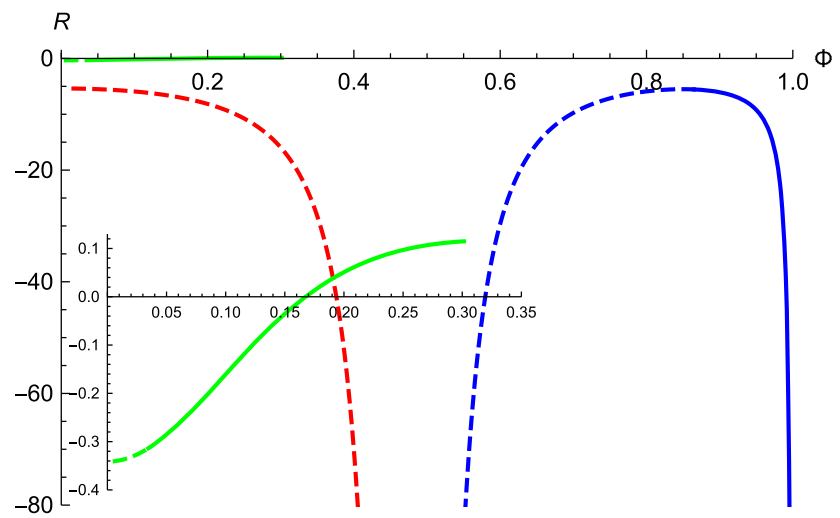

dashed lines represent metastable overcharged Small BH/undercharged Large $\mathrm{BH}$ while the red dashed lines denote unstable spinodal Intermediate BH. Left Column $T=0.2 . R$ is always negative. Right Column $T=0.24$. The inset shows that $R>0$ for the Large BH solution with a large enough potential

enough $\Phi$ can have $R>0$ in the AdS case while Large BH with a large enough $\Phi$ can have $R>0$ in the cavity case. Furthermore, the interactions among the microstructure of the saturated Large BH and Small BH on the coexisting line are always attractive in the cavity case. However in the AdS case, the interactions of the saturated Large BH and Small BH are repulsive and attractive, respectively, when $T \gtrsim 0.286$.

In this paper, we only considered different phases of RN black hole solutions in a canonical ensemble, in which the charge $Q$ of the system is fixed. Note that, when $Q=0$, the thermal space filled with neutral radiation is also a classical solution besides the black hole solution (i.e., a Schwarzschild black hole). In some region of the parameter space, the free energy of the thermal space can be less than that of the black hole solution, which leads to a first-order phase transition (e.g., the Hawking-Page phase transition in the AdS case). For charged black holes, one may wonder whether there is other solution of lower free energy with the same charge. One natural candidate is the thermal space filled with a charged

gas. However, the backreaction of the non-negligible charge of the gas on the geometry should be considered when the free energy is calculated. It is inspiring to explore how this possible equilibrium phase plays a role in the phase transitions of charged black holes in the future studies.

In summary, although the phase structure of a RN-AdS black hole and a RN black hole in a cavity is strikingly similar, we found that the thermodynamic geometry in the two cases behaves rather differently. It seems that the Ruppeiner invariant $R$ depends not only on what is inside the horizon, but also on the imposed boundary condition. Our results show that either $R$ encodes more than the nature of black hole microscopic properties, or there may be a connection between the black hole microstates and the boundary condition. It would be interesting to study how $R$ depends on the boundary condition in other black hole systems, which may shine further light on the connection between the internal microstructure of black holes and the boundary condition. 


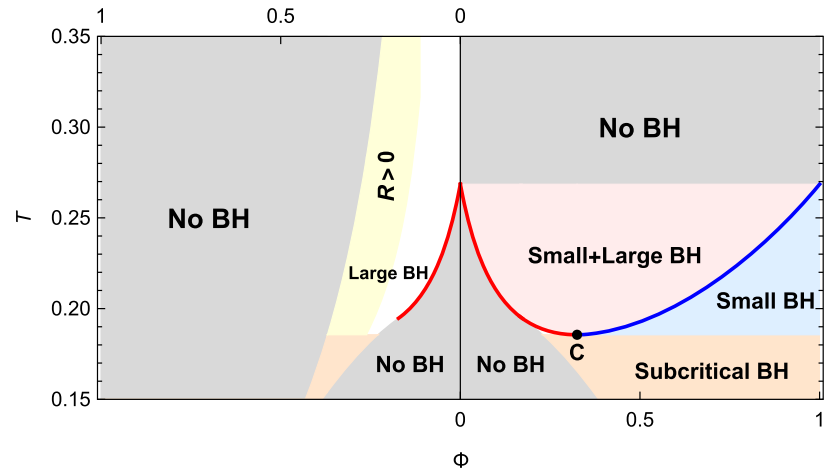

Fig. 9 Phase structure of a RN black hole in a cavity in the $\Phi-T$ diagram. The saturated Small BH (blue line) and Large BH (red line) are separated by the critical point (black dot). For a fixed value of $\Phi$, there exist two black hole solutions, which are displayed in the left and right frames, respectively. The pink region is the coexistence region of Small $\mathrm{BH}$ and Large $\mathrm{BH}$, and $R>0$ in the yellow region

Table 1 The Ruppeiner invariant $R$ for the black hole solutions in the AdS and cavity cases. Tabulated are the regions where $R>0$ and the possible divergences of $R$. BH denotes the single black hole solution when $Q>Q_{c}$. GSP strands for "globally stable phase"

\begin{tabular}{lll}
\hline Black hole solution & $R>0$ & $|R|$ divergence \\
\hline SBH in AdS & None & $T=0$ and $C_{Q}^{-1}=0$ \\
SBH in cavity & None & $C_{Q}^{-1}=0$ \\
LBH in AdS & Large $T$ & $C_{Q}^{-1}=0$ \\
LBH in cavity & None & $C_{Q}^{-1}=0$ \\
BH in AdS & Large $T$ & $T=0$ \\
BH in cavity & Large $T$ or small & None \\
& $T$ or all $T$ & \\
GSP in AdS & Large $T$ & $T=0$ and critical point \\
GSP in cavity & Large $Q$ & Critical point \\
\hline
\end{tabular}

Acknowledgements We are grateful to S. A. Hosseini Mansoori, Shuxuan Ying and Zhipeng Zhang for useful discussions and valuable comments. This work is supported in part by NSFC (Grant Nos. 11875196, 11375121 and 11005016).

Data Availability Statement This manuscript has no associated data or the data will not be deposited. [Authors' comment: The results of this paper are based on the theoretical derivation. We do not use any data in the calculations.]

Open Access This article is licensed under a Creative Commons Attribution 4.0 International License, which permits use, sharing, adaptation, distribution and reproduction in any medium or format, as long as you give appropriate credit to the original author(s) and the source, provide a link to the Creative Commons licence, and indicate if changes were made. The images or other third party material in this article are included in the article's Creative Commons licence, unless indicated otherwise in a credit line to the material. If material is not included in the article's Creative Commons licence and your intended use is not permitted by statutory regulation or exceeds the permitted use, you will need to obtain permission directly from the copyright holder. To view a copy of this licence, visit http://creativecomm ons.org/licenses/by/4.0/.

Funded by SCOAP ${ }^{3}$.

\section{References}

1. S.W. Hawking, Particle creation by black holes. Commun. Math. Phys. 43, 199 (1975). Erratum: [Commun. Math. Phys. 46, 206 (1976)]. https://doi.org/10.1007/BF02345020. https://doi.org/10. 1007/BF01608497

2. J.D. Bekenstein, Black holes and the second law. Lett. Nuovo Cim 4, 737 (1972). https://doi.org/10.1007/BF02757029

3. J.D. Bekenstein, Black holes and entropy. Phys. Rev. D 7, 2333 (1973). https://doi.org/10.1103/PhysRevD.7.2333

4. S.W. Hawking, D.N. Page, Thermodynamics of black holes in antide Sitter space. Commun. Math. Phys. 87, 577 (1983). https://doi. org/10.1007/BF01208266

5. J.M. Maldacena, The large $\mathrm{N}$ limit of superconformal field theories and supergravity. Int. J. Theor. Phys. 38, 1113 (1999). [Adv. Theor. Math. Phys. 2, 231 (1998)]. https://doi.org/10.1023/ A:1026654312961. https://doi.org/10.4310/ATMP.1998.v2.n2.a1 arXiv:hep-th/9711200

6. S.S. Gubser, I.R. Klebanov, A.M. Polyakov, Gauge theory correlators from noncritical string theory. Phys. Lett. B 428, 105 (1998). https://doi.org/10.1016/S0370-2693(98)00377-3. arXiv:hep-th/9802109

7. E. Witten, Anti-de Sitter space and holography. Adv. Theor. Math. Phys. 2, 253 (1998). https://doi.org/10.4310/ATMP.1998.v2.n2.a2. arXiv:hep-th/9802150

8. E. Witten, Anti-de Sitter space, thermal phase transition, and confinement in gauge theories. Adv. Theor. Math. Phys. 2, 505 (1998). https://doi.org/10.4310/ATMP.1998.v2.n3.a3. arXiv:hep-th/9803131

9. A. Chamblin, R. Emparan, C.V. Johnson, R.C. Myers, Charged AdS black holes and catastrophic holography. Phys. Rev. D 60, 064018 (1999). https://doi.org/10.1103/PhysRevD.60.064018. arXiv:hep-th/9902170

10. A. Chamblin, R. Emparan, C.V. Johnson, R.C. Myers, Holography, thermodynamics and fluctuations of charged AdS black holes. Phys. Rev. D 60, 104026 (1999). https://doi.org/10.1103/ PhysRevD.60.104026. arXiv:hep-th/9904197

11. M. M. Caldarelli, G. Cognola, D. Klemm, Thermodynamics of Kerr-Newman-AdS black holes and conformal field theories. Class. Quant. Gravit. 17, 399 (2000). https://doi.org/10.1088/ 0264-9381/17/2/310. arXiv:hep-th/9908022

12. R.G. Cai, Gauss-Bonnet black holes in AdS spaces. Phys. Rev. D 65, 084014 (2002). https://doi.org/10.1103/PhysRevD.65.084014. arXiv:hep-th/0109133

13. D. Kubiznak, R.B. Mann, P-V criticality of charged AdS black holes. JHEP 1207, 033 (2012). https://doi.org/10.1007/ JHEP07(2012)033. arXiv:1205.0559 [hep-th]

14. P. Wang, H. Wu, H. Yang, Thermodynamics and phase transitions of nonlinear electrodynamics black holes in an extended phase space. JCAP 1904, 052 (2019). https://doi.org/10.1088/1475-7516/2019/ 04/052. arXiv:1808.04506 [gr-qc]

15. P. Wang, $\mathrm{H}$. Wu, H. Yang, Thermodynamics of nonlinear electrodynamics black holes and the validity of weak cosmic censorship at charged particle absorption. Eur. Phys. J. C 79(7), 572 (2019). https://doi.org/10.1140/epjc/s10052-019-7090-z

16. C.S. Peca, J.P.S. Lemos, Thermodynamics of Reissner-Nordstrom anti-de Sitter black holes in the grand canonical ensemble. Phys. Rev. D 59, 124007 (1999). https://doi.org/10.1103/PhysRevD.59. 124007. arXiv:gr-qc/9805004

17. J.W. York Jr., Black hole thermodynamics and the Euclidean Einstein action. Phys. Rev. D 33, 2092 (1986). https://doi.org/10.1103/ PhysRevD.33.2092

18. H.W. Braden, J.D. Brown, B.F. Whiting, J.W. York Jr., Charged black hole in a grand canonical ensemble. Phys. Rev. D 42, 3376 (1990). https://doi.org/10.1103/PhysRevD.42.3376 
19. S. Carlip, S. Vaidya, Phase transitions and critical behavior for charged black holes. Class. Quant. Gravit. 20, 3827 (2003). https:// doi.org/10.1088/0264-9381/20/16/319. arXiv:gr-qc/0306054

20. A.P. Lundgren, Charged black hole in a canonical ensemble. Phys. Rev. D 77, 044014 (2008). https://doi.org/10.1103/PhysRevD.77. 044014. arXiv:gr-qc/0612119

21. J.X. Lu, S. Roy, Z. Xiao, Phase transitions and critical behavior of black branes in canonical ensemble. JHEP 1101, 133 (2011). https://doi.org/10.1007/JHEP01(2011)133. arXiv:1010.2068 [hep-th]

22. C. Wu, Z. Xiao, J. Xu, Bubbles and black branes in grand canonical ensemble. Phys. Rev. D 85, 044009 (2012). https://doi.org/10. 1103/PhysRevD.85.044009. arXiv:1108.1347 [hep-th]

23. J.X. Lu, R. Wei, J. Xu, The phase structure of black D1/D5 (F/NS5) system in canonical ensemble. JHEP 1212, 012 (2012). https://doi. org/10.1007/JHEP12(2012)012. arXiv:1210.0708 [hep-th]

24. J.X. Lu, R. Wei, Modulating the phase structure of black D6 branes in canonical ensemble. JHEP 1304, 100 (2013). https://doi.org/10. 1007/JHEP04(2013)100. arXiv:1301.1780 [hep-th]

25. D. Zhou, Z. Xiao, Phase structures of the black $\mathrm{D} p-\mathrm{D}(p+4)-$ brane system in various ensembles I: thermal stability. JHEP 1507, 134 (2015). https://doi.org/10.1007/JHEP07(2015)134. arXiv:1502.00261 [hep-th]

26. Z. Xiao, D. Zhou, Phase structures of the black D $p-\mathrm{D}(p+4)$ brane system in various ensembles II: electrical and thermodynamic stability. JHEP 1509, 028 (2015). https://doi.org/10.1007/ JHEP09(2015)028. arXiv:1507.02088 [hep-th]

27. P. Basu, C. Krishnan, P.N.B. Subramanian, Hairy Black Holes in a Box. JHEP 1611, 041 (2016). https://doi.org/10.1007/ JHEP11(2016)041. arXiv:1609.01208 [hep-th]

28. Y. Peng, B. Wang, Y. Liu, On the thermodynamics of the black hole and hairy black hole transitions in the asymptotically flat spacetime with a box. Eur. Phys. J. C 78(3), 176 (2018). https://doi.org/10. 1140/epjc/s10052-018-5652-0. arXiv:1708.01411 [hep-th]

29. Y. Peng, Studies of a general flat space/boson star transition model in a box through a language similar to holographic superconductors. JHEP 1707, 042 (2017). https://doi.org/10.1007/ JHEP07(2017)042. arXiv:1705.08694 [hep-th]

30. Y. Peng, Scalar field configurations supported by charged compact reflecting stars in a curved spacetime. Phys. Lett. B 780, 144 (2018). https://doi.org/10.1016/j.physletb.2018.02.068. arXiv:1801.02495 [gr-qc]

31. F. Simovic, R.B. Mann, Critical phenomena of charged de Sitter black holes in cavities. Class. Quant. Gravit. 36(1), 014002 (2019). https://doi.org/10.1088/1361-6382/aaf445. arXiv:1807.11875 [grqc]

32. P. Wang, H. Wu, H. Yang, Thermodynamics and phase transition of a nonlinear electrodynamics black hole in a cavity. JHEP 1907, 002 (2019). https://doi.org/10.1007/JHEP07(2019)002. arXiv:1901.06216 [gr-qc]

33. K. Liang, P. Wang, H. Wu, M. Yang, Phase structures and transitions of Born-Infeld black holes in a grand canonical ensemble. arXiv: 1907.00799 [gr-qc]

34. A. Strominger, C. Vafa, Microscopic origin of the BekensteinHawking entropy. Phys. Lett. B 379, 99 (1996). https://doi.org/10. 1016/0370-2693(96)00345-0. arXiv:hep-th/9601029

35. C.G. Callan, J.M. Maldacena, D-brane approach to black hole quantum mechanics. Nucl. Phys. B 472, 591 (1996). https://doi. org/10.1016/0550-3213(96)00225-8. arXiv:hep-th/9602043

36. R. Emparan, G.T. Horowitz, Microstates of a neutral black hole in M theory. Phys. Rev. Lett. 97, 141601 (2006). https://doi.org/10. 1103/PhysRevLett.97.141601. arXiv:hep-th/0607023

37. F. Weinhold, Metric geometry of equilibrium thermodynamics. J. Chem Phys. 63, 2479 (1975). Erratum: [J. Chem Phys. 63, 2484 (1975)]
38. G. Ruppeiner, Riemannian geometry in thermodynamic fluctuation theory. Rev. Mod. Phys. 67, 605 (1995). Erratum: [Rev. Mod. Phys. 68, 313 (1996)]. https://doi.org/10.1103/RevModPhys.67.605

39. H. Janyszek, R. Mrugala, Riemannian geometry and stability of ideal quantum gases. J. Phys. A Math. Gen. 23, 467 (1990)

40. H. Janyszek, R. Mrugala, Riemannian geometry and the thermodynamics of model magnetic systems. Phys. Rev. A 39, 6515 (1989). https://doi.org/10.1103/PhysRevA.39.6515

41. B. Mirza, H. Mohammadzadeh, Nonperturbative thermodynamic geometry of anyon gas. Phys. Rev. E 80, 011132 (2009)

42. S. Ferrara, G.W. Gibbons, R. Kallosh, Black holes and critical points in moduli space. Nucl. Phys. B 500, 75 (1997) https://doi. org/10.1016/S0550-3213(97)00324-6. arXiv:hep-th/9702103

43. J.E. Aman, N. Pidokrajt, Geometry of higher-dimensional black hole thermodynamics. Phys. Rev. D 73, 024017 (2006). https:// doi.org/10.1103/PhysRevD.73.024017. arXiv:hep-th/0510139

44. T. Sarkar, G. Sengupta, B. Nath Tiwari, On the thermodynamic geometry of BTZ black holes. JHEP 0611, 015 (2006). https://doi. org/10.1088/1126-6708/2006/11/015. arXiv:hep-th/0606084

45. J.Y. Shen, R.G. Cai, B. Wang, R.K. Su, Thermodynamic geometry and critical behavior of black holes. Int. J. Mod. Phys. A 22, 11 (2007). https://doi.org/10.1142/S0217751X07034064. arXiv:gr-qc/0512035

46. H. Quevedo, A. Sanchez, Geometrothermodynamics of asymptotically de Sitter black holes. JHEP 0809, 034 (2008). https://doi.org/ 10.1088/1126-6708/2008/09/034. arXiv:0805.3003 [hep-th]

47. R. Banerjee, S.K. Modak, S. Samanta, Second order phase transition and thermodynamic geometry in Kerr-AdS black hole. Phys. Rev. D 84, 064024 (2011). https://doi.org/10.1103/PhysRevD.84. 064024. arXiv:1005.4832 [hep-th]

48. D. Astefanesei, M.J. Rodriguez, S. Theisen, Thermodynamic instability of doubly spinning black objects. JHEP 1008, 046 (2010). https://doi.org/10.1007/JHEP08(2010)046. arXiv: 1003.2421 [hep-th]

49. S.W. Wei, Y.X. Liu, Y.Q. Wang, H. Guo, Thermodynamic geometry of black hole in the deformed Horava-Lifshitz Gravity. EPL 99, no. 2, 20004 (2012). https://doi.org/10.1209/0295-5075/99/ 20004. arXiv:1002.1550 [hep-th]

50. H. Liu, H. Lu, M. Luo, K.N. Shao, Thermodynamical metrics and black hole phase transitions. JHEP 1012, 054 (2010). https://doi. org/10.1007/JHEP12(2010)054. arXiv:1008.4482 [hep-th]

51. C. Niu, Y. Tian, X.N. Wu, Critical phenomena and thermodynamic geometry of RN-AdS black holes. Phys. Rev. D 85, 024017 (2012). https://doi.org/10.1103/PhysRevD.85.024017. arXiv:1104.3066 [hep-th]

52. S.W. Wei, Y.X. Liu, Critical phenomena and thermodynamic geometry of charged Gauss-Bonnet AdS black holes. Phys. Rev. D 87(4), 044014 (2013). https://doi.org/10.1103/PhysRevD.87. 044014. arXiv:1209.1707 [gr-qc]

53. R. Banerjee, B.R. Majhi, S. Samanta, Thermogeometric phase transition in a unified framework. Phys. Lett. B 767, 25 (2017). https:// doi.org/10.1016/j.physletb.2017.01.040. arXiv:1611.06701 [grqc]

54. T. Vetsov, Information geometry on the space of equilibrium states of black holes in higher derivative theories. Eur. Phys. J. C 79(1), 71 (2019). https://doi.org/10.1140/epjc/ s10052-019-6553-6. arXiv:1806.05011 [gr-qc]

55. H. Dimov, R.C. Rashkov, T. Vetsov, Thermodynamic information geometry and complexity growth of a warped AdS black hole and the warped $\mathrm{AdS}_{3} / \mathrm{CFT}_{2}$ correspondence. Phys. Rev. D 99(12), 126007 (2019). https://doi.org/10.1103/PhysRevD.99. 126007. arXiv:1902.02433 [hep-th]

56. K. Bhattacharya, S. Dey, B.R. Majhi, S. Samanta, General framework to study the extremal phase transition of black holes. Phys. Rev. D 99(12), 124047 (2019). https://doi.org/10.1103/PhysRevD. 99.124047. arXiv:1903.03434 [gr-qc] 
57. J.E. Aman, I. Bengtsson, N. Pidokrajt, Geometry of black hole thermodynamics. Gen. Relat. Gravit. 35, 1733 (2003). https://doi. org/10.1023/A:1026058111582. arXiv:gr-qc/0304015

58. B. Mirza, M. Zamani-Nasab, Ruppeiner geometry of RN black holes: flat or curved? JHEP 0706, 059 (2007). https://doi.org/10. 1088/1126-6708/2007/06/059. arXiv:0706.3450 [hep-th]

59. Z.M. Xu, B. Wu, W.L. Yang, The fine micro-thermal structures for the Reissner-Nordström black hole. arXiv:1910.03378 [gr-qc]

60. A. Sahay, T. Sarkar, G. Sengupta, On the thermodynamic geometry and critical phenomena of AdS black holes. JHEP 1007, 082 (2010). https://doi.org/10.1007/JHEP07(2010)082. arXiv: 1004.1625 [hep-th]

61. P. Chaturvedi, S. Mondal, G. Sengupta, Thermodynamic geometry of black holes in the canonical ensemble. Phys. Rev. D 98(8), 086016 (2018). https://doi.org/10.1103/PhysRevD.98. 086016. arXiv:1705.05002 [hep-th]

62. J.L. Zhang, R.G. Cai, H. Yu, Phase transition and thermodynamical geometry of Reissner-Nordstrom-AdS black holes in extended phase space. Phys. Rev. D 91(4), 044028 (2015). https://doi.org/ 10.1103/PhysRevD.91.044028. arXiv:1502.01428 [hep-th]

63. S.H. Hendi, A. Sheykhi, S. Panahiyan, B. Eslam Panah, Phase transition and thermodynamic geometry of Einstein-Maxwell-Dilaton black holes. Phys. Rev. D 92(6), 064028 (2015). https://doi.org/10. 1103/PhysRevD.92.064028. arXiv:1509.08593 [hep-th]

64. S.W. Wei, Y.X. Liu, Insight into the microscopic structure of an AdS black hole from a thermodynamical phase transition. Phys. Rev. Lett. 115(11), 111302 (2015). Erratum: [Phys. Rev. Lett. 116, no. 16, 169903 (2016)] https://doi.org/10.1103/PhysRevLett. 116.169903. https://doi.org/10.1103/PhysRevLett.115.111302. arXiv:1502.00386 [gr-qc]

65. A. Sahay, Restricted thermodynamic fluctuations and the Ruppeiner geometry of black holes. Phys. Rev. D 95(6), 064002 (2017). https://doi.org/10.1103/PhysRevD.95.064002. arXiv:1604.04181 [hep-th]

66. A. Dehyadegari, A. Sheykhi, A. Montakhab, Critical behavior and microscopic structure of charged AdS black holes via an alternative phase space. Phys. Lett. B 768, 235 (2017). https://doi.org/10. 1016/j.physletb.2017.02.064. arXiv:1607.05333 [gr-qc]

67. Y.G. Miao, Z.M. Xu, Microscopic structures and thermal stability of black holes conformally coupled to scalar fields in five dimensions. Nucl. Phys. B 942, 205 (2019). https://doi.org/10.1016/j. nuclphysb.2019.03.015. arXiv:1711.01757 [hep-th]

68. D. Li, S. Li, L.Q. Mi, Z.H. Li, Insight into black hole phase transition from parametric solutions. Phys. Rev. D 96(12), 124015 (2017). https://doi.org/10.1103/PhysRevD.96.124015

69. Y.Z. Du, R. Zhao, L.C. Zhang, Microstructure and continuous phase transition of the Gauss-Bonnet AdS black hole. arXiv:1901.07932 [hep-th]

70. X.Y. Guo, H.F. Li, L.C. Zhang, R. Zhao, Microstructure and continuous phase transition of a Reissner-Nordstrom-AdS black hole. Phys. Rev. D 100(6), 064036 (2019). https://doi.org/10.1103/ PhysRevD.100.064036. arXiv:1901.04703 [gr-qc]
71. S.W. Wei, Y.X. Liu, R.B. Mann, Repulsive interactions and universal properties of charged black hole microstructures. Phys. Rev. Lett. 123(7), 071103 (2019). https://doi.org/10.1103/PhysRevLett. 123.071103. arXiv: 1906.10840 [gr-qc]

72. S.W. Wei, Y.X. Liu, R.B. Mann, Ruppeiner geometry, phase transitions, and the microstructure of charged AdS black holes. arXiv: 1909.03887 [gr-qc]

73. S.W. Wei, Y.X. Liu, Intriguing microstructures of five-dimensional neutral Gauss-Bonnet AdS black hole. arXiv:1910.04528 [gr-qc]

74. D. Kastor, S. Ray, J. Traschen, Enthalpy and the mechanics of AdS black holes. Class. Quant. Gravit. 26, 195011 (2009). https://doi. org/10.1088/0264-9381/26/19/195011. arXiv:0904.2765 [hep-th]

75. B.P. Dolan, Pressure and volume in the first law of black hole thermodynamics. Class. Quant. Gravit. 28, 235017 (2011). https:// doi.org/10.1088/0264-9381/28/23/235017. arXiv:1106.6260 [grqc]

76. S. Gunasekaran, R.B. Mann, D. Kubiznak, Extended phase space thermodynamics for charged and rotating black holes and BornInfeld vacuum polarization. JHEP 1211, 110 (2012). https://doi. org/10.1007/JHEP11(2012)110. arXiv:1208.6251 [hep-th]

77. L. McGough, M. Mezei, H. Verlinde, Moving the CFT into the bulk with $T \bar{T}$. JHEP 1804, 010 (2018). https://doi.org/10.1007/ JHEP04(2018)010. arXiv:1611.03470 [hep-th]

78. P. Wang, $\mathrm{H}$. Wu, H. Yang, The dual geometries of $T \bar{T}$ deformed $\mathrm{CFT}_{2}$ and highly excited states of $\mathrm{CFT}_{2}$. arXiv:1811.07758 [hepth]

79. P. Chaturvedi, A. Das, G. Sengupta, Thermodynamic geometry and phase transitions of dyonic charged AdS black holes. Eur. Phys. J. C 77(2), 110 (2017). https://doi.org/10.1140/epjc/ s10052-017-4678-z. arXiv:1412.3880 [hep-th]

80. S.A.H. Mansoori, B. Mirza, Correspondence of phase transition points and singularities of thermodynamic geometry of black holes. Eur. Phys. J. C 74(99), 2681 (2014). https://doi.org/10.1140/epjc/ s10052-013-2681-6. arXiv:1308.1543 [gr-qc]

81. S.A.H. Mansoori, B. Mirza, M. Fazel, Hessian matrix, specific heats, Nambu brackets, and thermodynamic geometry. JHEP 1504, 115 (2015). https://doi.org/10.1007/JHEP04(2015)115. arXiv:1411.2582 [gr-qc]

82. S.A.H. Mansoori, B. Mirza, Geometrothermodynamics as a singular conformal thermodynamic geometry. Phys. Lett. B 799, 135040 (2019). https://doi.org/10.1016/j.physletb.2019.135040. arXiv:1905.01733 [gr-qc] 